\title{
Imaging of gene expression in vivo with photoacoustic tomography
}

\section{Li Li, Roger J. Zemp, Gina Lungu, George Stoica, Lihong V. Wang}

Li Li, Roger J. Zemp, Gina Lungu, George Stoica, Lihong V. Wang, "Imaging of gene expression in vivo with photoacoustic tomography," Proc. SPIE 6086, Photons Plus Ultrasound: Imaging and Sensing 2006: The Seventh Conference on Biomedical Thermoacoustics, Optoacoustics, and Acoustooptics, 608608 (6 March 2006); doi: 10.1117/12.646322

SPIE. Event: SPIE BiOS, 2006, San Jose, California, United States 


\title{
Imaging of Gene Expression in vivo with Photoacoustic Tomography
}

\author{
$\mathrm{Li} \mathrm{Li}^{\mathrm{a}}$, Roger J. Zempa ${ }^{\mathrm{a}}$, Gina Lungu ${ }^{\mathrm{b}}$, George Stoica ${ }^{\mathrm{b}}$, and Lihong V. Wang ${ }^{\mathrm{a}, *}$ \\ a. Optical Imaging Laboratory, Department of Biomedical Engineering, \\ b. Department of Pathobiology, \\ Texas A\&M University, College Station, Texas, 77843-3120
}

\begin{abstract}
In the post-genomic era, there is an increasing interest in visualizing the expression of functional genes in vivo. With the assistance of the reporter gene technique, various imaging modalities have been adopted for this purpose. In vivo gene expression imaging promises to provide biologists with a powerful tool for deepening our understanding of developmental biology, expanding our knowledge of the genetic basis of disease, and advancing the development of medicine. In this paper, we demonstrate the feasibility of imaging gene expression with photoacoustic imaging, which offers unique absorption contrast with ultrasonic resolution in vivo. We mark tumors in rats with the lacZ reporter gene. The lacZ gene encodes an enzyme $\beta$-galactosidase, which yields a dark blue product when acting on a colorimetric assay called X-gal. Photoacoustic tomography is able to visualize the presence of this blue product clearly in the far red range of the optical spectrum. Considering how many staining methods are used in traditional biology, we believe that photoacoustic techniques will revolutionize the field of molecular imaging. The further development of reporter gene systems with high absorbing products in the NIR region is needed.
\end{abstract}

Keywords: photoacoustic, molecular imaging, gene expression, reporter gene, lacZ

\section{INTRODUCTION}

Thanks to the Human Genome Project ${ }^{1}$, we now have a book of human genetic codes in hand. However, in the post-genome era, the bigger challenge of deciphering these codes is still ahead. As a branch of the emerging field of molecule imaging, a series of imaging modalities have recently been exploited to image the activities of functional genes in vivo, including Positron Emission Tomography (PET), Single Photon Emission Tomography (SPECT) and Magnetic Resonance Imaging (MRI). ${ }^{2}$ These techniques have a significant advantage, compared to the widely used ex-vivo methods like DNA micro-array, because they have the capabilty to visualize the expression of genes in their natural environment as a dynamic process. For our purposes, an extraneous "reporter gene" is usually incorporated into the genome of cells or a particular animal model. The protein product of this gene can provide the required contrast for a certain imaging modality either directly or by acting on a biochemical assay. It is called a "reporter gene" because its expression is related to the function of a promoter or enhancer, the activity of another endogenous gene of interest, or the presence of marked cells, etc. Recently, optical methods have attracted interest in this field because of their intrinsic rich contrast, high sensitivity, low cost, and ease of operation. Some successful paradigms include fluorescence and bioluminescence. ${ }^{3}$

The main challenge to these optical techniques is the high scattering in biological tissue. In this paper, we propose, for the first time to our knowledge, photoacoustic tomography (PAT) as a promising candidate for imaging gene expression in vivo, which we believe will open a new horizon in this field. PAT relies on optical absorption. The photoacoustic waves generated by laser-induced thermo-elastic expansion in

* Corresponding author. Email address: $\underline{\text { lwang@,tamu.edu }}$ 
absorbing subjects are detected by ultrasonic transducers under certain geometries. The absorbing structures are then mapped by coherent reconstruction. In addition to being a non-invasive imaging modality, PAT combines the merits of high sensitivity to absorbing contrast and high ultrasonic resolution at the sub-millimeter level. G. Ku et al. ${ }^{4}$ have achieved a penetration depth of several centimeters in tissue phantoms. Success has been achieved in depicting both healthy and malignant vasculature in the brains of small animals, like rats and mice, which are the popular animal models for molecular imaging. ${ }^{5,6}$ Functional imaging of the level of the oxygen saturation in blood is also possible with photoacoustic techniques ${ }^{5,7}$. Desirable contrast agents provide high optical absorption, preferably in the far-red or near-infrared range, where light has the deepest penetration in tissue. Some of these, such as indocyanine green (ICG) ${ }^{8}$, poly(ethylene glycol)-coated nanoshells, ${ }^{9}$ have been used in in vivo applications

We chose the lacZ reporter gene, a classical bacterial reporter gene, for our first attempt at imaging gene expression using PAT. It encodes an enzyme, $\beta$-galactosidase, which cleaves glycosidic linkages in certain sugars, like lactose. Only a few studies have reported to see the expression of the lacZ gene in vivo. The first was done using MRI by Louie by introducing a novel $\beta$-galactosidase-cleavable paramagnetic contrast agent into an embryo of Xenopus at the two-cell stage. ${ }^{10}$ Recently, Tung et al used a fluorescence dye, DDAOG to image the expression of lacZ using planar fluorescence in mice. ${ }^{11}$ We used a sensitive chromogenic assay, containing primarily a lactose-like sugar called 5-bromo-4-chloro-3-indolyl- $\beta$-Dgalactoside (X-gal), to delineate the expression pattern of the lacZ gene. A stable dark blue product was generated after the X-gal was cleaved. ${ }^{12}$ The lacZ/X-gal strategy was previously used widely in genetics studies as an important histochemical method in dissected tissue samples or embryos.

\section{MEASUREMENT OF ABSORPTION SPECTRUM}

To choose the proper wavelength for imaging, we measured the molar extinction spectrum of the blue product, which is reported on here for the first time to our knowledge. $9 \mathrm{~L} / \mathrm{lacZ}$ gliosarchoma tumor cells (cultured from cell line from American Type Culture Collection) were washed and centrifuged with cell lysate. The supernatant containing $\beta$-galactosidase was divided into 5 cuvettes with $120 \mu 1$ each. Then they were diluted by $600 \mu 1$ of water. To the first four cuvettes, we added $20 \mu \mathrm{l}$ of X-gal solution (Fermentas, Inc) with concentrations of $2,0.4,0.2$, and $0.04 \mathrm{mg} / \mathrm{mL}$ respectively. $20 \mu 1$ of water was added to the last cuvette for a baseline. After allowing enough time for reaction, the absorbance of these solutions was measured using a spectrometer (USB2000, Ocean Optics, Inc.) with a broadband light source (SOL-R 150DC). Because the small amount of X-gal is used, we assumed all of X-gal molecules were cleaved, and thus the concentration of blue product can be calculated. Molar extinction coefficients at certain wavelengths were obtained from the slope of the linear fitting between the absorbance and the concentration of the blue product. In Figure 1, we compared the measured molar extinction spectrum of the blue product with blood which has the oxygen saturation $\left(\mathrm{SO}_{2}\right)$ level at $90 \%$. The blue product has absorption peak, which is stronger than blood, around $620-690 \mathrm{~nm}$. This is at the far red range of the optical spectrum which means it potentially enables deep penetration.

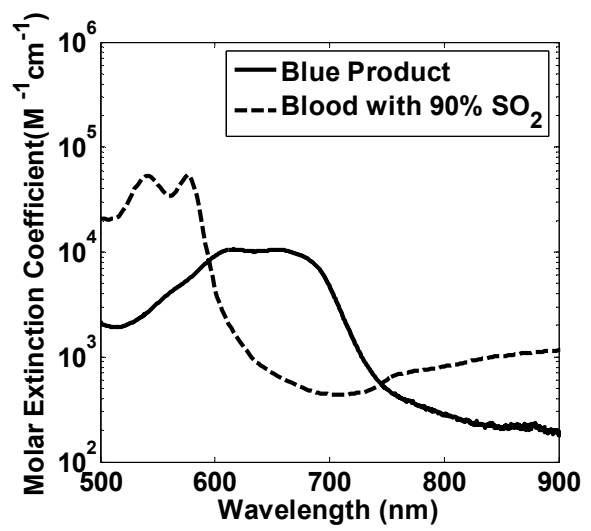

Figure 1. Comparison of molar extinction spectrum of the blue product and blood with $90 \% \mathrm{SO}_{2}$. 


\section{ANIMAL EXPERIMENTS}

\subsection{Cells and animals}

9L/LacZ gliosarchoma tumor cells were cultured until they were $\sim 70 \%$ confluent. About 5 million cells were inoculated subcutaneously under the scalps of Sprague Dawley rats and left to grow until a small tumor was present. Hair on the head was removed before imaging. Animals were kept under anaesthesia by a gas anesthesia machine (E-Z Anesthesia, Euthanex Corp.) using isoflurane during the experiments. The blood oxygenation and heart rates were monitored using a pulse-oximeter (NONIN 8600V). $25 \mu$ of X-gal (Fermentas, Inc.) was injected subcutaneously near the tumor. A control injection of X-gal was injected in a non-tumor location, usually in a symmetric position on the other side of the head to avoid any confusion arising from the possible existence of endogenous $\beta$-galactosidase. Another photoacoustic image was taken afterwards.

\subsection{System Setup}

The scheme of our PAT system setup is shown in Figure 2. A Q-switched Nd:YAG laser (Quantel Brilliant B) at 532nm was used to pump a dye laser (Continuum ND6000). We chose laser dye DCM (Exciton Inc., Dayton, OH), which provided us a broad range of wavelengths from 608 to $689 \mathrm{~nm}$. The output of the dye laser was directed using prisms. Before it reached the animal, a concave lens and ground glass were employed to broaden the illumination. The laser energy was measured to be about $5 \mathrm{~mJ} / \mathrm{cm}^{2}$ at the imaging plane. An ultrasonic transducer (V323-SU, Panametrics-NDT) with a center frequency of $2.25 \mathrm{MHz}$ was scanned by a customized mechanical gantry system, driven by a stepper motor, around a circle to receive the outgoing photoacoustic waves at 240 step locations. Signals were later amplified by pre-amplifiers made from broadband RF amplifiers (ZFL500, Mini-circuits Inc.), digitized and averaged at each scan position using a digital oscilloscope (TDS5034, Tektronix), and then transferred to a PC. To compensate for the fluctuation, a photo-diode (DET110, ThorLabs, Inc.) was used to record the relative incident laser power at each position. Signals were triggered by Q-switch synchronization. A program in LabVIEW was used to control the stepper motor, change the wavelengths from the dye laser, and acquire the data.

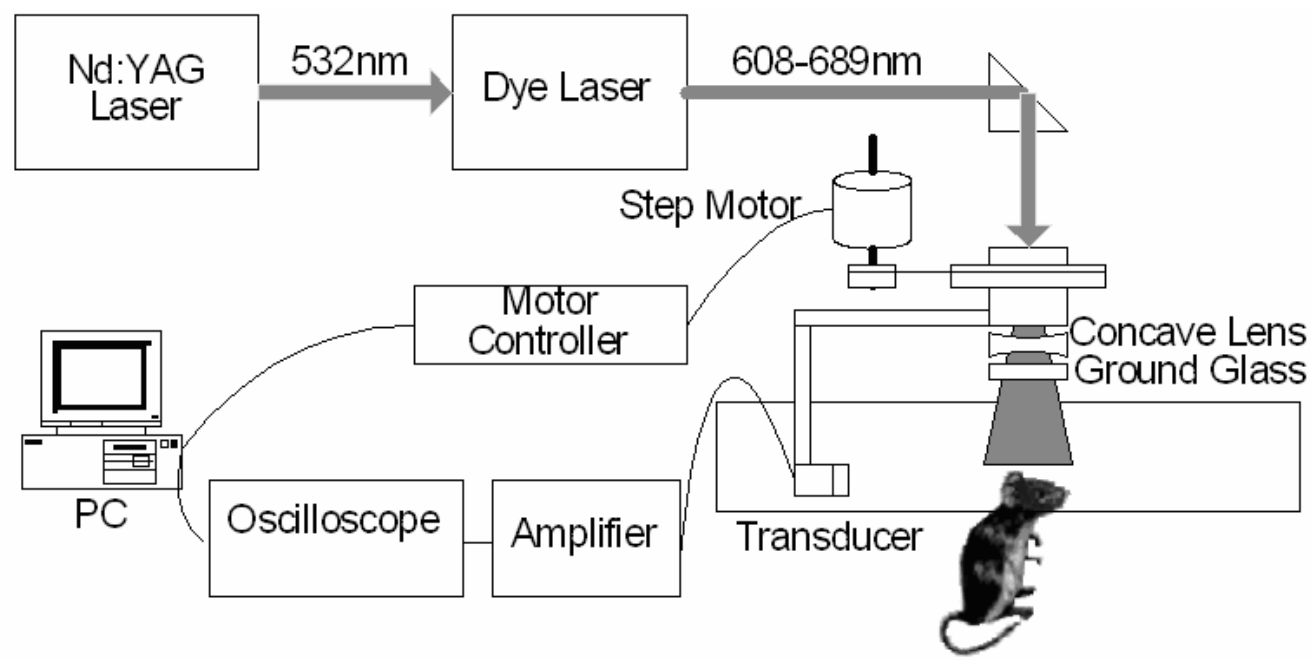

Figure 2. Scheme of the photoacoustic tomography system. 


\subsection{Image Reconstruction}

Images were reconstructed following a modified back-projection algorithm ${ }^{13}$ from the collected sinogram, which represents the spatial distribution of the absorption coefficients, under assumptions of uniform illumination and homogenous mechanical properties in soft tissues.

\subsection{Results}

Figure 3 shows a typical result of photoacoustic imaging at $635 \mathrm{~nm}$. Figure 3(a) shows the top view of the intact rat head before imaging. The tumor in this case is flat and is hardly visible from outside. The marked region is where we imaged. Other parts of the head are covered by a white cloth to protect the eyes of the animal and reduce interference signals. Figure 3(b) shows the flipped skin corresponding to the dashed rectangular region. The black region is dark blue in color, which represents the region of the tumor with the blue product as a result of the expression of the lacZ gene. Figure 3(c) is a photoacoustic image taken before the injection of the $\mathrm{X}$-gal. The vasculature of the brain is visible. The relatively strong middle line is the image of the median fissure. No obvious sign of a tumor appears. Figure 3(d) shows the image taken after the X-gal injection. The tumor shows up dramatically and overwhelms the signal from blood. Its shape is similar to the histological view.
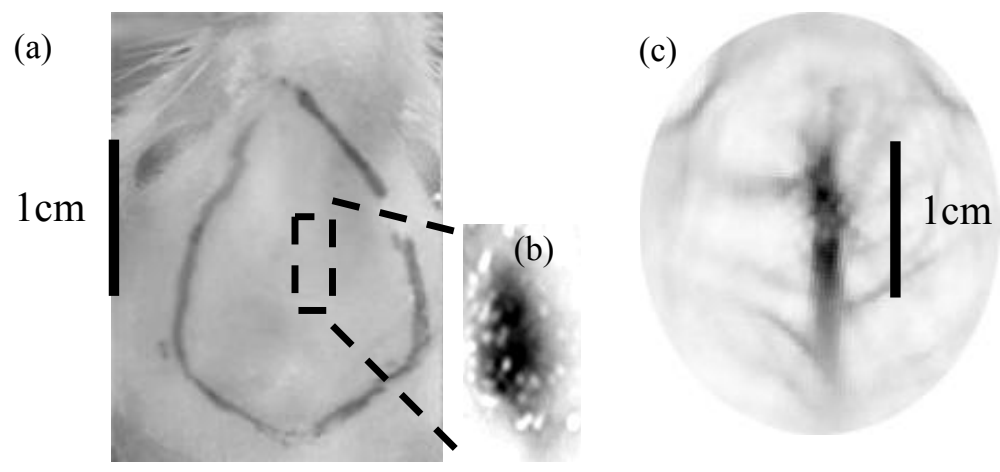

(d)

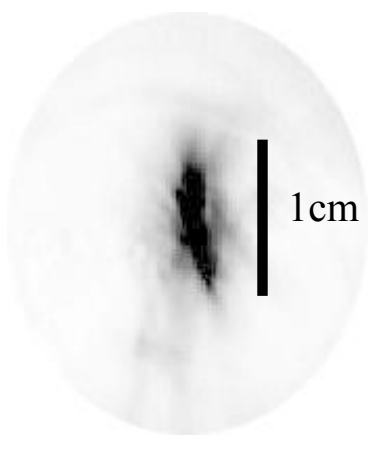

Figure 3. Results from single-wavelength photoacoustic tomography at $635 \mathrm{~nm}$. (a) Top view of the intact rat head. (b) Flipped skin of the dashed rectangular region; the tumor region is dark blue in color. (c) Photoacoustic image before the injection of X-gal. (d) Photoacoustic image after the injection of X-gal.

\section{DISCUSSION}

Because of the dominant absorption of the blue product, the expression of the lacZ gene is clearly imaged at $635 \mathrm{~nm}$ in our animal model. This implies the high efficiency of the generation of the blue product. An important advantage of the enzyme-based reporter gene systems (e.g. lacZ) over others (e.g. Green Fluoresence Protein) is their intrinsic amplification mechanism. A molecule of the expressed product is capable of acting on a huge number of probing molecules, and, thus, signal amplification is achieved. Potentially, by exploiting the spectroscopic information of optical absorption, a multi-wavelength technique with careful calibration will be able to separate the contributions from different absorbers, which will be especially helpful when we have low levels of expression.

The spatial resolution of the current system was estimated by imaging a phantom with two crossed hairs. The smallest separable distance between the centers of the two hairs in the image is about $400 \mu \mathrm{m}$. The sensitivity is estimated by taking A-line measurements of a small tube at $650 \mathrm{~nm}$, filled by a solution of blue product at a concentration of 0.27 Molar, embedded $5 \mathrm{~mm}$ deep in chicken tissue. The SNR was calculated to be around 75 . This means we had a sensitivity better than $3.6 \mu$ Molar for the blue product. The sensitivity for the expressed products, $\beta$-galactosidase, should be much higher than this due to the amplification mechanism. The resolution can be improved by adopting high-frequency transducers, at the cost of losing sensitivity. Due to the slow pulse repetition rate (PRF) of the laser at $10 \mathrm{~Hz}$ and the 
mechanical scanning scheme, it took about 25 minutes to achieve one single-wavelength image. The speed can be improved by employing a high PRF laser and transducer array techniques.

There are some limitations to in vivo application of X-gal. It appears to cause skin irritation occasionally. The dynamics of the reaction needs oxidation ${ }^{14}$ and is poorly understood in vivo. Currently, we deliver Xgal by local injection. Systemic delivery of X-gal through the tail vein does not seem efficient in our animal model. There are a huge number of staining methods used in traditional biology, and an enormous number of pigments existing in nature. Further development of new reporter gene systems providing absorption contrast can be expected. It is preferable that the absorption of the reaction products have peaks in the far red and near-infrared regions of the optical spectrum, where light can penetrate deep into the tissues.

Progress in techniques for imaging gene expression will advance gene therapy where monitoring the delivery efficiency of therapeutic genes is a big challenge.

\section{CONCLUSION}

In summary, we have demonstrated the feasibility of imaging gene expression by photoacoustic imaging. The expression of lacZ is visualized in the far red range of optical spectrum by photoacoustic tomography. We believe this technique will open new horizons in this field and lead to the future development of new absorption-based reporter gene systems.

\section{ACKNOWLEDGEMENT}

We are grateful to Sergio Similache, and Ovidiu Craciun for assistance with animal handling. This research is funded in part by the National Institutes of Health grants R01 N846214 (BRP) and R01 EB000712.

\section{REFERENCES}

1. International Human Genome Sequencing Consortium, "Finishing the euchromatic sequence of the human genome," Nature, 431, 931 - 945 (2004).

2. T. F. Massoud, and S. S. Gambhir, "Molecular imaging in living subjects: seeing fundamental biological processes in a new light," Genes and Development, 17, 545-580 (2003).

3. B. Christoph, and R. Weissleder, "In vivo imaging of gene expression: MR and optical technologies," Acad. Radiol., 8(1), 15-23 (2001).

4. G. $\mathrm{Ku}$, and L. V. Wang, "Deeply penetrating photoacoustic tomography in biological tissues enhanced with an optical contrast agent," Opt. Lett., 30(5), 507-509 (2005).

5. X. Wang, Y. Pang, G. Ku, X. Xie, G. Stoica, and L. V. Wang, "Non-invasive laser-induced photoacoustic tomography for structural and functional imaging of the brain in vivo," Nat. Biotech. 21(7), 803-806 (2003).

6. G. Ku, X. Wang, X. Xie, G. Stoica and L. V. Wang, "Imaging of tumor angiogenesis in rat brains in vivo by photoacoustic tomography," Appl. Opt., 44(5), 770-775 (2005).

7. R. O. Esenaliev, I. V. Larina, D. J. Deyo, M. Motamedi, and D.S. Prough, "Optoacoustic technique for noninvasivemonitoring of blood oxygenation: a feasibility study," Appl. Opt. 41(22), 4722-4731 (2002).

8. X. Wang, G. Ku, M. A. Wegiel, D. J. Bornhop, G. Stoica and L. V. Wang, "Non-invasive photoacoustic angiography of animal brains in vivo with NIR light and an optical contrast agent," Opt. Lett. 29(7), 730732 (2004).

9. Y. Wang, X. Xie, X. Wang, G. Ku, K. L. Gill, D. P. O’Neal, G. Stoica and L. V. Wang, "Photoacoustic tomography of a nanoshell contrast agent in the in vivo rat brain," Nano Lett., 4(9), 1689-1692 (2004).

10. A. Y. Louie, M. M. Huber, and E.T. Ahrens, "In vivo visualization of gene expression using magnetic resonance imaging," Nat. Biotech., 18, 321-325 (2000).

11. C. H. Tung, Q. Zeng, K. Shah, D. E. Kim, D. Schellingerhout, and R. Weissleder, "In vivo imaging of $\beta$-galactosidase activity using far red fluorescent switch," Cancer Res. 64(5), 1579-83 (2004). 
12. H. Lodish, A. Berk, S.L. Zipursky, P. Matsudaira, D. Baltimore, and J. Darnell, "Molecular Cell Biology, "W.H. Freeman and Company, New York (2000).

13. M. Xu, Y. Xu and L. V. Wang, "Time-domain reconstruction algorithms and numerical simulations for thermoacoustic tomography in various geometries," IEEE Trans. Med. Imaging, 50(9), 1086-1099 (2003). 14. S. J. Holt, and P. W. Sadler, "Studies in enzyme cytochemistry III. relationships between solubility, molecular association and structure in indigoid dyes," Proc R Soc Lond B Biol Sci. 148(933), 495-505 (1958). 